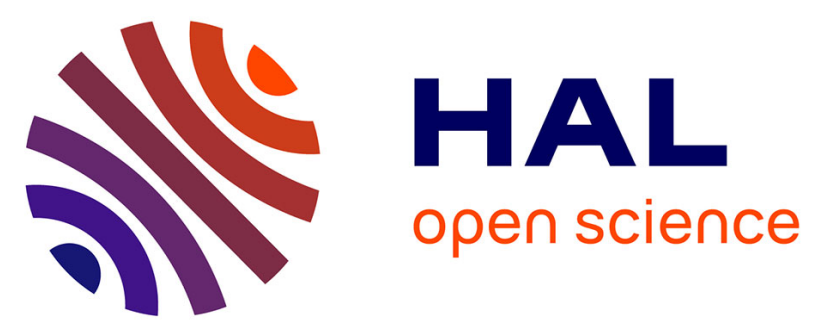

\title{
Automatic event-level textual emotion sensing using mutual action histogram between entities
}

Cheng-Yu Lu, Shian-Hua Lin, Jen-Chang Liu, Samuel Cruz-Lara, Jen-Shin Hong

\section{- To cite this version:}

Cheng-Yu Lu, Shian-Hua Lin, Jen-Chang Liu, Samuel Cruz-Lara, Jen-Shin Hong. Automatic eventlevel textual emotion sensing using mutual action histogram between entities. Expert Systems with Applications, 2009, 37 (2), pp.1643-1653. 10.1016/j.eswa.2009.06.099 . inria-00426567

\section{HAL Id: inria-00426567 https://hal.inria.fr/inria-00426567}

Submitted on 27 Oct 2009

HAL is a multi-disciplinary open access archive for the deposit and dissemination of scientific research documents, whether they are published or not. The documents may come from teaching and research institutions in France or abroad, or from public or private research centers.
L'archive ouverte pluridisciplinaire HAL, est destinée au dépôt et à la diffusion de documents scientifiques de niveau recherche, publiés ou non, émanant des établissements d'enseignement et de recherche français ou étrangers, des laboratoires publics ou privés. 
Elsevier Editorial System(tm) for Expert Systems With Applications Manuscript Draft

Manuscript Number:

Title: Automatic Event-Level Textual Emotion Sensing Using Mutual Action Histogram between Entities

Article Type: Full Length Article

Keywords: emotion sensing; web text mining; semantic role labeling; affect recognition

Corresponding Author: Dr. Shian-Hua Lin, Ph.D.

Corresponding Author's Institution: Department of Computer Science and Information Engineering First Author: Cheng-Yu Lu

Order of Authors: Cheng-Yu Lu; Shian-Hua Lin; Jen-Chang Liu; Samuel Cruz-Lara; Jen-Shin Hong 


\title{
Automatic Event-Level Textual Emotion Sensing Using Mutual Action Histogram between Entities
}

\author{
Cheng-Yu Lu ${ }^{1,2}$, Shian-Hua Lin ${ }^{1}{ }^{*}$, Jen-Chang Liu ${ }^{1}$, Samuel Cruz-Lara ${ }^{2}$, Jen-Shin Hong ${ }^{1}$ \\ ${ }^{1}$ Dep. of Computer Science and Information Engineering, National Chi Nan University, Taiwan \\ ${ }^{2}$ LORIA - University of Nancy, France \\ * Corresponding Author, shlin@ncnu.edu.tw
}

\begin{abstract}
Automatic emotion sensing in textual data is crucial for the development of intelligent interfaces in many interactive computer applications. This paper describes a high-precision, knowledgebase-independent approach for automatic emotion sensing for the subjects of events embedded within sentences. The proposed approach is based on the probability distribution of common mutual actions between the subject and the object of an event. We have incorporated web-based text mining and semantic role labeling techniques, together with a number of reference entity pairs and hand-crafted emotion generation rules to realize an event emotion detection system. The evaluation outcome reveals a satisfactory result with about $85 \%$ accuracy for detecting the positive, negative and neutral emotions.
\end{abstract}

Keywords: emotion sensing, web text mining, semantic role labeling, affect recognition

\section{Emotion sensing from textual data}

Research on interface agents shows that a system's capacity for emotional interactions can makes the agents valuable [Bates 1994]. Aiming at enabling computers to express and to recognize emotions, emerging technological advances are inspiring the field of research on "affective computing" [Picard 1997]. Since many computer user interfaces today are textually-based, the automatic emotion recognition from textual data plays an important role in the design of intelligent user interfaces that are more natural and user-friendly.

In the past, many studies have been conducted to automatically detect a user's affective states from textual data. Some using "keyword-spotting" techniques [Elliott 1992], but the results are not satisfactory. Keyword-spotting approach apparently can't apply to sentences without clearly-defined affective keywords. A number of studies applied emotion theories to determine emotions of interactive agents in intelligent systems [Dyer 1987; Bartneck 2002]. In those approaches a variety of hand-crafted emotion models based on psychological theories (particularly those of Ortony, Clore, and Collins [Ortony et al. 1988]) were employed to specify how interactive events, agents and objects are appraised according to individual's goals, standards and attitudes. At this stage, emotion sensing based on an emotion theory, is 
only applicable in interactive systems where the interactive events can be precisely defined, enumerated and automatically detected. Because of the thorough nature of this approach, its application in free-texts requires a detailed understanding and analysis of the text which is rather beyond the reach of current natural language processing techniques.

[Liu et al. 2003] reported an approach to detect sentence-level emotion based on a large-scale common sense knowledgebase, ConceptNet. The approach uses real world knowledge about the inherent affective nature of everyday situations (such as "getting into a car accident") to classify sentences into basic emotion categories. In the initial stage, concepts in the ConceptNet with clearly defined affective keywords were automatically annotated with desired basic emotion categories. Then the emotion for other concepts with semantic relationships to the affectively annotated concepts are assigned automatically based on certain emotion propagation models. The accuracy of such emotion propagation process has not yet been investigated. In our opinion, the restricted coverage of the concepts and relationships in ConceptNet seriously limits the use of such approach in real life applications.

[Shaikh et al. 2007] recently developed a linguistic tool "SenseNet" to detect polarity values of word, phrase, and sentence-level textual data. The approach uses WordNet [Fellbaum 1998] as a linguistic corpus. Polarity values for adjectives and adverbs in WordNet were manually annotated. The polarity of a verb is calculated via some hand-crafted equations that count positive and negative senses from the definitions in WordNet. The polarity of a noun is assigned based on the related verbs obtained from relationships recorded in ConceptNet. For instance, "ticket" is connected to the following verbs "allow", "access", "get", "provide", and "represent" in ConceptNet. Thus the polarity of "ticket" is assigned as the average value of these verbs. One major problem is that since the data in ConceptNet contains many misspelled words, false concepts, and overly-specific data [Smith and Thomas 2004], the correctness of polarity value of many concepts in SenseNet is questionable. Moreover, SenseNet can only handle concepts existing in WordNet, so the concepts not included in WordNet cannot be processed.

[Wu et al. 2006] recently proposed a novel approach for sentence-level emotion detection based on the semantic labels (SLs) and attributes (ATTs) of entities of a sentence. To distinguish the emotions "happy" and "unhappy", the SLs are manually classified into three categories, Active SLs (e.g. obtain, reach, lost, hinder), Negative SLs (e.g. no, never), and Transitive SLs (e.g. finally, but, fortunately). ATTs of an entity are automatically obtained by using WordNet as the lexical resource. For example, in the sentence "I lost my money", the emotion would be unhappy due to the verb "lost" (an Active SL) and the attributes of "money" (such as wealth and property). Wu carried out his work in two phases, training phase and testing phase. In 
the training phase, a collection of sentences tagged with corresponding emotion states are used as the training set. The SLs and ATTs of these sentences are automatically extracted and then processed to obtain a variety of emotion associate rules (EARs) that associate a particular emotion states with patterns of the SLs and ATTs. In the testing phase, a target sentence is processed in the same manner to obtain its SLs and ATTs before feeding into an emotion classifier. The emotion of the target sentence is assigned based on the similarity comparison of the SLs and ATTs to the sets in the EARs. An evaluation was conducted by using a small-scale corpus of a collection of college students' daily conversations.

Consequently, the major issues of emotion sensing that might limit the performance and applicability of this approach in wider contexts include: (1) the need of affective-annotated sentences as training samples, and (2) the use of attributes (ATTs) as the sentence-level emotion-invoking ingredients. First, the proposed approach requires a sufficient large number of emotion-annotated sentences that are not frequently available promptly within other domains. Furthermore, in many real life situations, the "emotion-invoking" factors of an event may not always be possible to be explicitly represented by the attributes (ATTs) of the event participants. Emotions determined only with the attributes recorded in WordNet may often cause erroneous results. For example, although a "rat" and a "squirrel" have quite a number of similar or even identical attributes, rodent and mammal, as annotated in WordNet, emotions evoked by both entities (the rat and the squirrel) are totally different. Even worse, in real-life applications, event participants in a sentence often cover a wide-spectrum of modern or domain-specific terms which may not be included in formal lexical resources such as WordNet. For the example "Wii", the newest home video game console released by Nintendo, which could often be mentioned in daily conversations or news articles these days, is unfortunately not yet included in the current version of WordNet. It is essential for a really universal and robust emotion detection system to handle these situations.

As a first step for our endeavor towards a robust emotion sensing engine from free-texts using web mining approaches, this study proposes a novel approach for detecting emotion of an individual "event" embedded in English sentences such as "a student failed in the examination", "a girl saw a diamond", "a cat was chased by a dog", "a mouse encountered a cat", etc. We adopt the "common mutual actions" between the event participants as the major cue to determine the event-level emotions. In a real-life application when the emotions for free-text sentences are to be detected automatically, the subject, verb, and object of an event embedded in a free-text sentence can be obtained using Semantic Role Labeling (SRL) techniques. A general overview of the state-of-the-art SRL techniques has been discussed fully in [Gildea and Jurafsky 2002; Pradhan et al. 2003; Carreras and Marquez 2005]. Simply speaking, in any sentence, a verb (predicate) dominates an event. The syntactic 
arguments of a verb are usually associated with the participants of the event. A "semantic role" is the relationship of a syntactic argument with the verb. One commonly used scheme for specifying semantic roles is PropBank annotation [Dang and Palmer 2005; Punyakanok et al. 2004; Punyakanok et al. 2005]. In PropBank annotations, the arguments of a verb are labeled sequentially from ARG0 to ARG5, where ARG0 is usually the subject of a transitive verb; ARG1 is a direct object, etc. A variety of adjunctive arguments, such as ARGM-LOC for locatives, and ARGM-TMP for temporal information, are also tagged. As an illustrative example, the set of semantic roles for the sentence, "I saw a girl in the park this morning" based on the PropBank style markup, can be presented as:

[ARG0 I] [Target saw] [ARG1 a girl] [ARGM-LOC in the park] [ARGM-TMP this morning]

SRL techniques can be applied to automatically identify the semantic roles of a sentence. However, automatically tagging the semantic roles with high precision is difficult since an event can often be referred to, by a variety of lexical items with different syntactic realizations. In the literature survey, a number of studies have proposed different methodologies for this purpose, such as [Gildea and Jurafsky 2002; Pradhan et al. 2003; Koomen et al. 2005]. These methodologies have yielded quite accurate results with about $80 \%$ precision on ARG0, ARG1, and $70 \%$ on ARGM-LOC, ARGM-TMP [Carreras and Marquez 2005; Pradhan et al. 2004]. Given the reasonable accuracy of current state-of-the-art SRL techniques, the automatically parsing of subject, object, and verb in a free-text sentence for the emotion-sensing applications is satisfactory.

In the following sections, the underlying principles of our approach will be elaborated.

\section{Emotion sensing based on Mutual Action Histograms (MAHs)}

This section describes the underlying principles and detail processes of our methodology for automatically sensing emotions of events embedded in textual sentences. We first present here a typical scenario to illustrate the steps to achieve our goal. In any English sentence, the verb of a sentence typically indicates a particular "action" performed by one event participant to the other participant. For example, in the sentence "The girl saw a viper snake", the action is a "sighting action" with two entities: "the girl" as the subject and "a viper snake" as the object. While we often intuitively assume that the girl would often be terrified while meeting a viper snake, but how can a computer understand this?

Let us investigate why a girl would usually be terrified when she sees a viper snake. One way to reason is that the snake "usually" performs certain undesirable actions 
(e.g. bite, paralyze, attack, or kill) but "seldom" performs desirable actions (e.g. love, feed, or supply) on a girl. Contrarily, a baby would usually be happy to see his/her mother since his/her mother "usually" perform desirable actions on the baby. The real-life probability distribution of such "mutual actions" between a subject and an object is termed as the "Mutual Action Histogram (MAH)" in this paper. In practice, knowing the MAH between the subject and the object in a specific event would allow a computer to reasonably guess the emotion invoked. For example, if the MAH between a vampire and a girl is close to that between a snake and a girl, the emotion of a girl when she saw a vampire should most likely similar to that of a girl when she saw a snake. As compared to the relevant work by [Wu et al. 2006], our approach adopts the mutual actions between the event participants instead of using their attributes as the features to determine the emotions.

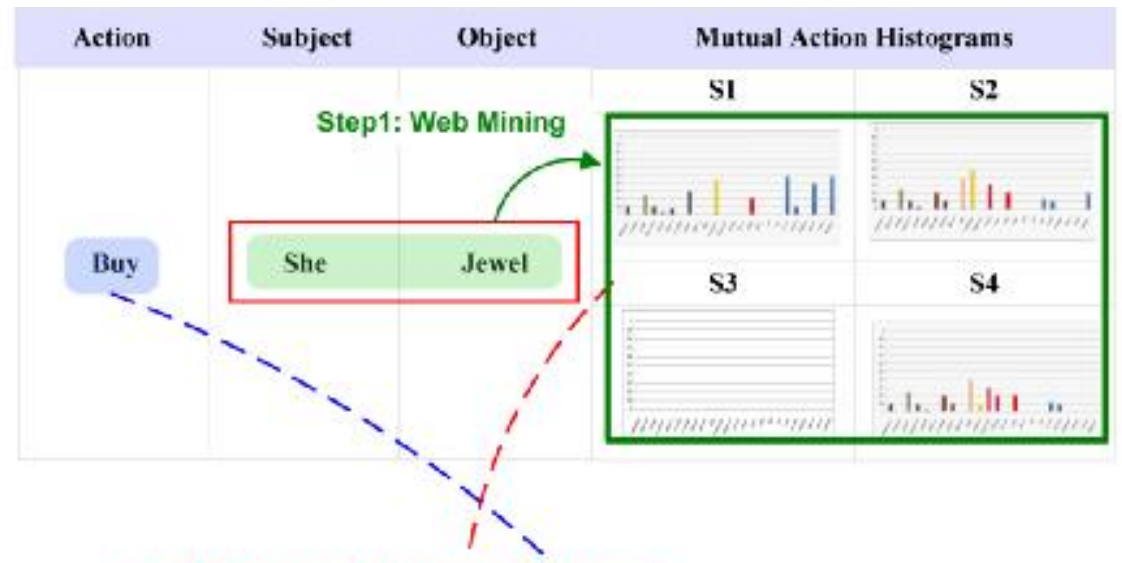

Step2: Matching Reference Entity Pairs

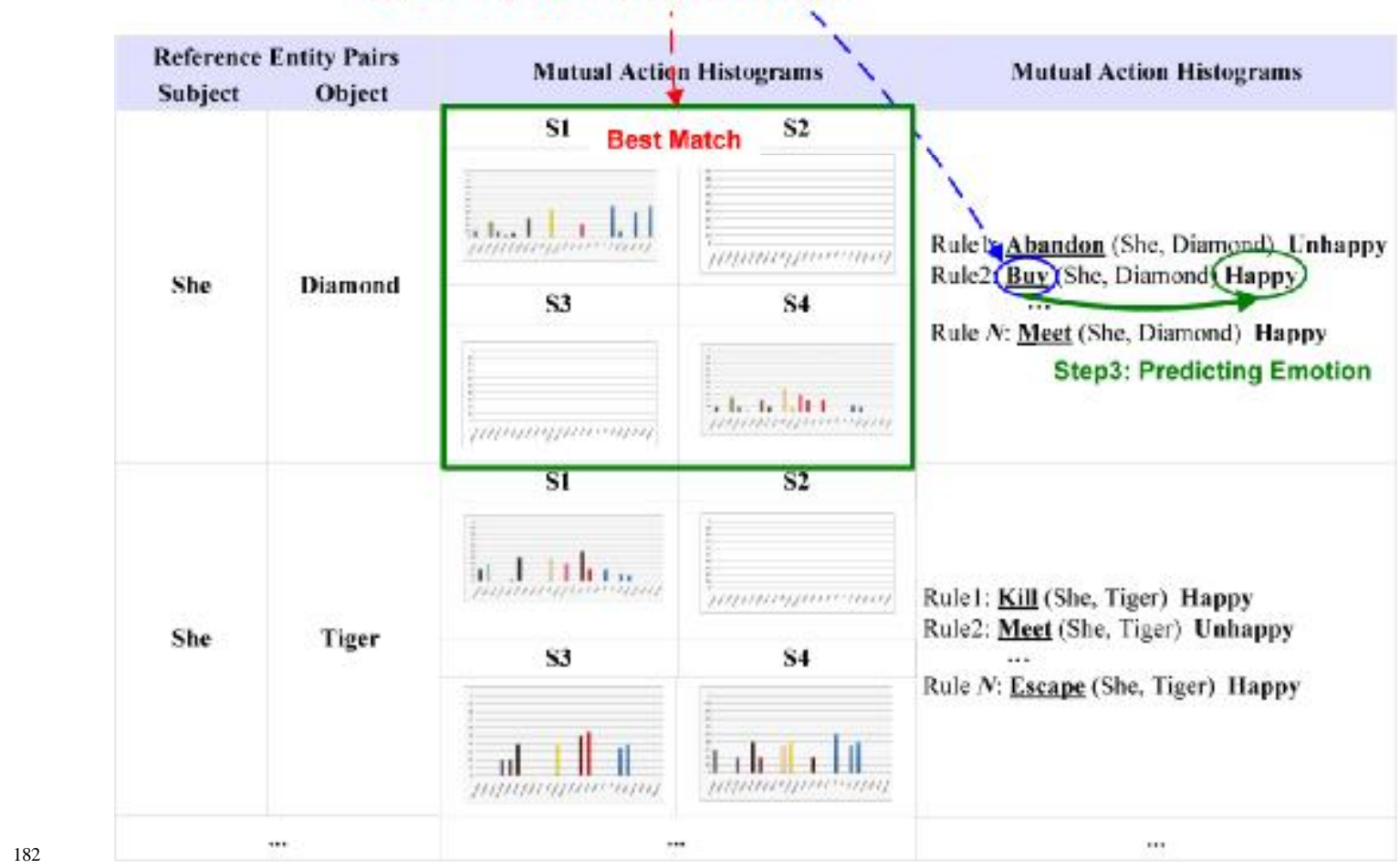

Figure 1. Illustrative diagram for the proposed approach. 
Based on the above analysis, it appears that the success of our approach for emotion detection would hinge on the ability of a computer to automatically retrieve the MAHs between two entities that participate in an event. While people may intuitively believe that MAHs between entities are difficult or impossible to obtain without extensive human annotations, we resort to web-based text mining that fortunately provide a convenient solution to achieve our goal. We introduce the overall flow diagram for our proposed emotion detection engine, as shown in Figure 1 below. First, in the training phase, we select a number of entity pairs (subject-object, e.g. girl-spider, girl-diamond, etc.) as the Reference Entity Pairs (RE-Pairs). For each RE-Pair, the MAH is obtained using web-based text mining techniques. Then for each RE-Pair, possible emotion-invoking events that often occur between the two entities are enumerated and manually assigned with emotions to form a set of "Emotion Generation Rules" (EGRs). In the predicting (or testing) phase (emotion sensing stage), the subject and object of a target event, termed as the Target Entity Pair (TE-Pair), in a sentence is automatically recognized using semantic role labeling techniques. The MAH of the TE-Pair is obtained using web-based text mining techniques to search and extract TE-Pair sentences from search engines. The emotion of the target event is assigned based on the EGRs of the RE-Pairs with best match on the MAHs to that of the TE-Pair. In the following, the underlying principles and detail operations of the proposed framework will be elaborated.

\subsection{Web-based text mining for MAHs between entities}

We apply web-based text mining techniques to retrieve mutual actions between two given entities. In the past, studies based on variations of text mining approaches have been investigated to retrieve different types of knowledge (e.g. [Gildea et al. 2006; Etzioni et al. 2005; Girju et al. 2006]). Given the vast amount of textual data available on the Web, we believe that the actions of two given entities described in web pages shall give a roughly normalized distribution of their common interactions. In the text mining approach, given a target event with two entities (the subject and target objects), the system analyzes the mutual actions between both entities by collecting large amount of emotion-invoking sentences from the Web. Therefore, hand-crafted "lexico-syntactic patterns" are generated and submitted to search engines for accumulating sentences from web pages within the search results. We borrow the glossary "lexico-syntactic patterns (LSPs)" from [Hearst 1992], in which a LSP indicates a particular semantic relationship between two entities.

In this paper, a set of effective lexico-syntactic patterns are formulated to describe emotion-invoking sentences. These sentences can be written either in "passive" or "active" style and the subject/target entities can be put in active/passive roles. 
Consequently, given an Event (E1, A, E2), with an action $\mathbf{A}$ and two entities E1 and E2, four different styles of LSP sentences are automatically generated as followings:

ü S1 (E1-Active-E2): (e.g., "She saw a snake.”)

ü S2 (E2-Passive-E1): (e.g., "The snake was killed by her.”)

ü S3 (E2-Active-E1): (e.g., “A snake bites her.”)

ü S4 (E1-Passive-E2): (e.g., "She was bitten by a snake.”)

The following examples illustrate the process for obtaining the mutual actions between two given entities. Considering an attempt to retrieve sentences that describe the mutual actions between "female" and "snake", typically intuitive syntactic patterns can be applied to formulate following web queries:

ü S1: "she * a snake",

ü S2: "snake was * by her",

ü S3: "snakes * her", and

ü S4: "she was * by the snake".

In case there are insufficient collected sentences by using these primitive patterns, it is possible to extend the patterns by considering variations of the verb tenses (e.g. "she was * by the snake" in S4), plural forms (e.g. "snakes were * by her" in S2), and articles (e.g. "she * the snake"). With these query strings, we typically accumulate a large number of raw sentences from the snippets of web search results. To effectively and efficiently compare the MAHs among entities pairs, the collected action verbs will be categorized and normalized for estimating the MAHs of each writing styles respectively.

\subsubsection{Verb categorization}

In practice, the matching for the MAHs between the TE-Pair and RE-Pair are based on a coarse-grained verb categorization. We collected about 800 popular verbs from Yahoo! Online Dictionary ${ }^{1}$. Given an entity pair of an event, semantically similar verbs typically invoke a similar emotion to the subject. Thus, these collected verbs are first categorized into 87 groups of synonyms (refer to Appendix 1). Within these verb groups, we manually determine whether a given verb group is "emotion-invoking" or not. If a verb of a given event often causes a certain emotion to the subject or object, it is considered as emotion-invoking. For example, if E1 frequently "attacks" E2, it is

${ }^{1}$ http://tw.dictionary.yahoo.com/ 
270

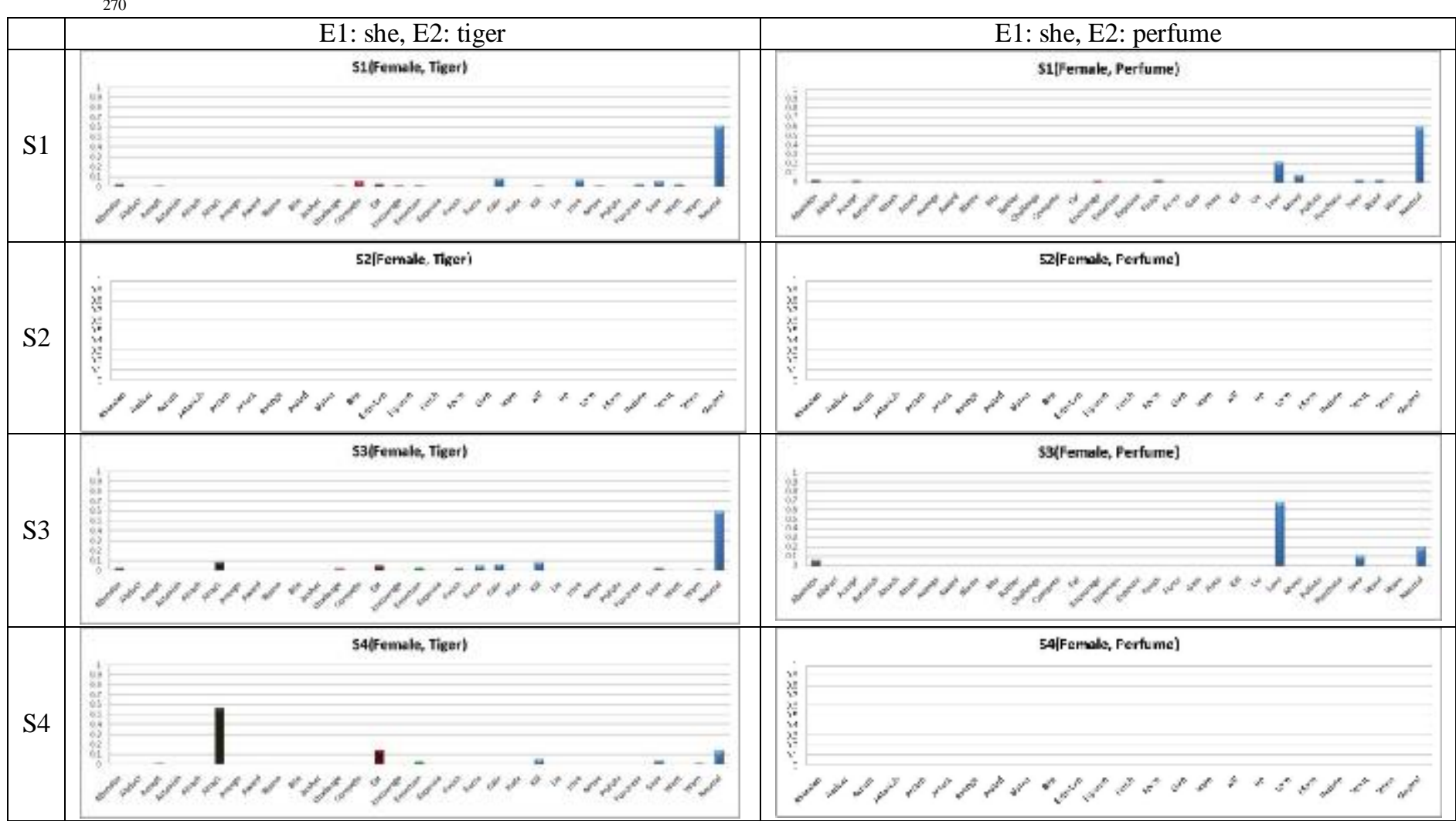
emotion-invoking verb. These emotion-invoking verbs are used as the main features to predict the emotion of the subject or object of an event. Based on this principle, 30 emotion-invoking verb groups are manually selected by experts, including abandon, abduct, accept, astonish, attach, attack, avenge, award, blame, bite, bother, challenge, compete, eat, encourage, entertain, espouse, finish, force, gain, hate, kill, lie, move, pollute, purchase, save, want and warn. Verbs other than those identified as emotion-invoking verbs are grouped as "neutral" verbs. Consequently, experts manually assign 800 verbs into 31 verb groups.

Figure 2: Mutual Action Histograms for two entity pairs.

For each sentence style, among all the sentences extracted from crawled pages, we count all frequencies based on verbs appearing within the 31 verb groups. Thus, the 4-style histogram is specialized into four 31-group histograms, S1 - S4. In each style histogram, each bin is normalized by the total occurrences of 31 verb groups; the histogram therefore presents the probability distribution of 31 verb groups in the style template. Figure 2 shows the histograms of two examples entity pairs: (she, tiger) and (she, perfume). Notably, a female frequently "loves" perfume (S3) but is frequently "attacked" by a tiger (S4). Overall, both cases have significantly different histograms.

naturally that $\mathbf{E 1}$ is an unpleasant object to $\mathbf{E 2}$ and thus "attack" is considered as an 
Following research issue is finding the best matching between the MAHs of a TE-Pair with the RE-pairs so that the query emotion can be predicted by applying the best matching on MAHs.

\subsection{Reference Entity Pairs (RE-Pairs) with Emotion Generation Rules (EGRs)}

We select a number of RE-Pairs appearing in daily emotional events and manually assign emotions to those events to form a set of EGRs. The MAHs between RE-Pairs are obtained by using the web-based text mining approach described above. Obviously, the interaction between two entities of a RE-Pair is widely described in web pages so that the MAH would reliably represent the un-biased distribution of mutual actions between them. To widely cover different emotion-invoking scenarios, the RE-Pair set needs to take care of all the emotion categories for the training performance. Also, all actions defined in the verb groups should be considered. However, given a RE-Pair, only few actions are used to describe the events of the RE-Pair. That is many actions are zero-frequency in the RE-Pair's MAHs as we can see in Figure 2. Consequently for each RE-Pair, a set of EGRs are manually constructed for all verb groups, in which zero-frequency verbs are labeled as "N/A". Appendix 2 gives a list of EGRs for an example RE-Pair, "she" and "diamond ring". Rule 1 says that the emotion of a female when she "abandons" a diamond ring is typically unhappy. Rule 4 says a female is typically happy when she "accepts" a diamond ring.

\subsection{Matching MAHs among RE-Pairs}

In the emotion recognition stage, the emotion of the subject in a target event is assigned based on the similarity comparison between the MAHs of the TE-Pair (i.e. the event participants) with those of the RE-Pairs. The emotion of the subject in a target event is based on the RE-Pairs with the most similar MAH to that of the TE-Pair. For instance, given the target event, "a girl meets a wolf", the MAH that a wolf frequently acts on a girl is similar to that of the RE-Pair, "female" and "snake". The emotion of the girl is inferred as "unhappy" according to the "meet" EGRs of the RE-Pair, "female" and "snake."

Conventionally, comparisons of histograms can be achieved by using "Mean Square Error (MSE)" or "KL divergence" algorithms [Kullback and Leibler 1951]. We applied the MSE approach in this study since it is more computational efficient. Let the 4-style 31-verb histograms of a TE-Pair be $H_{T}=\left\{t_{i}: i=1 \ldots 124\right\}$, and the 4-tyle histograms of a RE-Pair be $H_{R}=\left\{r_{i}: i=1 \ldots 124\right\}$. The $L_{l}$ distance is adopted to compute the dissimilarity between the 4-style histograms of the TE-Pair and the 
4-style histograms of the RE-Pairs. $L_{l}$ distance has been used to compare histograms in many applications, such as image retrieval [Rubner et al. 2000]. The $L_{l}$ distance between two histograms is defined as:

$$
d_{L_{1}}\left(H_{T}, H_{R}\right)=\sum_{i}\left|t_{i}-r_{i}\right|
$$

The RE-Pair with the minimum $L_{l}$ distance to the TE-Pair is considered to have the most similar relationship between entities as the target event. For example, the entity pair (she, purse) is matched to (she, diamond) with minimum $L_{1}$ distance. It is apparent that two entity pairs have a similar emotion relationship, while (she, purse) and (she, snake) have a larger distance in the entity-relationship space. After matching the target pair to one RE-pair, the emotion of the subject in a target event is inferred from the EGRs of the matched RE-Pair.

\section{Evaluations}

To evaluate the performance of the proposed methodology we have conducted the following evaluation experiments. First, a number of entity pairs, covering "pleasant", "unpleasant", and "neutral" objects, are selected as RE-Pairs. MAHs of RE-Pairs are automatically measured based on the web text mining approach that analyzes sentences from large amount of web pages. Then, three researchers label each sentence with one of three emotional categories: "positive (happy)", "negative (unhappy)", and "neutral". These data form the training set of the system.

The testing data are also collected from sentences that describe daily events. These testing data are labeled by the manpower different from the training data to reserve the objectivity of evaluation. Details of training, testing, evaluation and observation processes are illustrated in the following sections.

\subsection{The Training Phase}

In the training phase, the subject "she" and following objects touched in the daily life are selected to form 11 RE-Pairs.

ü Pleasant objects: diamond ring, purse, new dress, prince;

ü Unpleasant objects: tiger, thief, spider, bug;

ü Neutral objects: table, tissue, book. 
For each RE-Pair, the web text mining processes of generating the MAHs (124-dimensions: four styles of 31 verb groups) and emotion generation rules (EGRs) of the RE-Pair are summarize as followings:

$\ddot{u}$ The general web crawler collects emotion-invoking web pages by submitting queries (e.g. "she * diamond ring") to search engines and following links within search results to crawl more pages, as described in section 2.1.

ü According to these collected web pages, each sentence is extracted and parsed by the SRL tool to identify the objects and verbs of the event. We use the publicly available SRL tool, ASSERT [Pradhan et al. 2004], to perform the task. Although only the query "she * diamond ring" was issued to the search engine, more RE-Pairs are also collected after the SRL parsing process. For example, other emotion-invoking sentences with subject "she" and verb group "buy" contain objects, such as "chicken nuggets", “i-pod”, "ps2”, etc. These novel objects will be considered as testing objects in the testing phase.

ü Based on the RE-Pair (e.g. she and diamond ring) and 31 verb groups, the statistical information is used to generate the MAH of the RE-Pair as shown in the aforementioned Figure 2.

$\ddot{u}$ Then, the emotions of events corresponding to the RE-Pair and 31 verb groups are manually labeled as: happy, unhappy, neutral, or N/A, as shown in Appendix 2. The RE-Pair and one verb group determine the emotion of the event forms the emotion generation rule (EGR). For example, the RE-Pair (she, diamond ring) with EGRs corresponding to "actions" was manually labeled as shown in Appendix 2. The rule 1 presents the knowledge: (she, diamond ring) AND "abandon" imply the "unhappy" emotion. The rule 4 indicates: (she, diamond ring) AND "accept" imply "happy". The rule 2 is labeled as "N/A" since people seldom describe (she, diamond ring) AND "abduct" in the same event. Each EGR is determined based on an agreement reached by three researchers.

Consequently, the EGR set consists of 341 rules associated to the emotions of all events generated from 11 RE-Pairs and 31 verb groups.

\subsection{The Testing Phase}

In the testing phase (or predicting phase), using "she" as the subject, various objects and verbs (actions) that a female widely encounters in her daily life are selected to be TE-Pairs (roughly 120 or so). By randomly combining verbs with the TE-Pairs, we 
efficiently generated thousands sentences as candidate events of the testing set. For example, sentences about daily life, such as "she saw a car accident", "she plays a Wii", "she buys a hamburger", etc. are automatically generated as events in this way. Obviously, the authors have manually precluded various unreasonable events (e.g. "she drinks a computer") for the experiment. Consequently, 600 sentences with equal-distribution on three emotion categories (roughly labeled by the authors) are employed in the evaluation experiment, as shown in Table 1.

Table 1: number of sentences for evaluation

\begin{tabular}{|l|l|l|l|}
\hline Emotion Categories & Positive & Negative & Neutral \\
\hline \# of composed sentences (events) & 200 & 200 & 200 \\
\hline
\end{tabular}

To objectively evaluate the testing result, the testing events are labeled by the manpower different from that of the training set. We recruit 10 graduate students (mostly graduate students majoring in computer science) to assess these 600 events. Every assessor is requested to judge the emotion for each of the six hundred events as one of the "Positive", "Negative", and "Neutral" emotions. The ground-truth emotion of an event is determined by the majority voting from the answers of assessors. Among the 600 events, 529 events receive more than 5 consistent votes (the half of votes) among the 10 assessors, in which the 529 events include 184/175/170 positive/negative/neutral sentences. Human assessors do not reach consistent emotion judgments on many events, such as "she changes her dress" and "she rides a bicycle". Only 161 events receive more than 9 consistent votes among the 10 assessors. This implies that there is a certain degree of inconsistency (uncertainty) in the emotional evaluations.

The testing set is shown in Appendix 3. The web mining system automatically generates lexico-syntactic patterns based on the subject "she" with those objects and verbs listed in Appendix 3. Then, the same crawling and analyzing processes are applied to these events for measuring MAHs of TE-Pairs. Given the MAH of a TE-Pair, the matching process illustrated in section 2.3 is applied to find the best matched MAH from the RE-Pair set. The EGRs (rule set) of the matched RE-Pair is triggered by the action (verb) of the event, the emotion of the event is therefore predicted based on the emotion category labeled in the fired EGR.

\subsection{Experiment Results}

The performance of the proposed emotion detection system is measured by the precision of correct predictions. Based on the events and emotions labeled in section 3.2 , the ground-truth testing sets are dynamically determined based on the threshold of majority votes reached by assessors. Accordingly, the precision rate is defined as: 


$$
\text { precision }=\frac{\# \text { of correctly answered events by system }}{\# \text { of events with the majority vote }>n} .
$$

The evaluation results are shown in Table 2. For the case with the threshold of majority vote $(n>5)$, the precision rate is about $77.5 \%$. As the threshold $n$ increases, the precision rate also increases accordingly. The highest precision rate $90.0 \%$ is achieved at $n=9$. This indicates that the system is able to correctly predict emotions of events that human assessors tend to have consistent opinions on those emotions. In general, the system achieves a high precision rate $85 \%$ by averaging all cases of majority votes $(n=5-9)$.

Table 2: Evaluation results of the proposed emotion detection system

\begin{tabular}{|c|c|c|c|c|c|c|c|c|c|}
\hline $\begin{array}{l}\text { Majority } \\
\text { votes }(n)\end{array}$ & $\begin{array}{l}\text { \# of } \\
\text { Sentences }\end{array}$ & Positive & Negative & Neutral & Precision & $\begin{array}{c}\text { \# of sentences } \\
\text { (correct) }\end{array}$ & $\begin{array}{l}\text { Positive } \\
\text { (correct) }\end{array}$ & $\begin{array}{l}\text { Negative } \\
\text { (correct) }\end{array}$ & $\begin{array}{l}\text { Neutral } \\
\text { (correct) }\end{array}$ \\
\hline 5 & 529 & 184 & 175 & 170 & $77.5 \%$ & 410 & 130 & 146 & 134 \\
\hline 6 & 380 & 141 & 115 & 124 & $81.0 \%$ & 308 & 98 & 102 & 108 \\
\hline 7 & 303 & 103 & 102 & 98 & $87.1 \%$ & 264 & 79 & 92 & 93 \\
\hline 8 & 183 & 54 & 66 & 63 & $88.5 \%$ & 162 & 40 & 61 & 61 \\
\hline 9 & 161 & 46 & 57 & 58 & $90.0 \%$ & 145 & 35 & 53 & 57 \\
\hline
\end{tabular}

\subsection{Observations}

Based on the web text mining approach, many events with novel object nouns are correctly predicted. For example, tested sentences with the lexical-syntactic pattern "she bought a * yesterday" are correctly predicted for novel objects, such as "Wii", "i-Phone", “i-Pod", "PS2", "Mercedes S600", "Rolex", etc. Those novel nouns are usually found in daily conversations but absent in the dictionary or the common sense knowledgebase so that emotions of sentences contain novel nouns are hard to be identified by knowledge-based approaches. Consequently, the web text mining method is adaptive to the rapidly changed web environment.

In previous emotion-detection systems, some wrong predictions are raised from modifiers (e.g. adjective "luxury" or adverb adjective "pretty good") that are often used to modify noun phrases of TE-Pair objects within general sentences. Apparently, the modifier may change the meaning of a noun phrase so that the emotion of the sentence is also influenced. For instance, two opposite sentences "she bought a nice car" and "she bought a broken car" are decorated with opposite modifiers "nice" and "broken", respectively. Therefore, for the same object "car" and verb "buy", both sentences are labeled with positive and negative emotions, respectively. The advantage of the proposed methodology is that a modifier to the noun phrase can be completely identified as an object so that "nice car" or "broken car" are individually processed by the web mining approach and are correctly matched with MAHs of RE-Pairs. For example, the S1 template of the TE-Pair (she, nice car) shows female usually loves/purchases a nice car and dominates the matching process toward to the 
RE-Pair (she, purse). Similarly, the TE-Pair (she, broken car) is matched with the RE-Pair (she, thief) since the S1 template of (she, broken car) presents female usually abandons/dislikes a broken car as that of (she, thief).

According to the experiment results, the highest precision rate is $90.0 \%$ with the threshold of majority vote 9 , i.e. $90 \%$ assessors have the same emotion judgment. In this test, there are 11, 4 and 1 sentences mismatched with manually labeled emotion categories of "positive", "negative" and "neutral", respectively. Some of the incorrectly predicted sentences are depicted below and observations of these error predictions are also discussed for seeking further improvements.

The problems of wrong predictions result largely from the polysemy entities that have multiple senses corresponding to different emotion interpretations. For instance, the TE-Pairs (she, jaguar) and (she, giant) do not match with the correct (or relevant) RE-Pairs, because "jaguar" and "giant" are usually regarded as the famous brands that produce vehicles and bikes, respectively. So assessors labeled both entities as "Happy". However, "jaguar" is a panther and often performs several unpleasant actions (e.g. attack, kill, etc.). After analyzing from web pages, MAHs of both TE-Pairs tend to clarify general meanings so that the system inferred the "negative" emotion of "she meets a jaguar" from that of "she meets a tiger".

As for the semantic problem about the subject "she", females generally have positive feeling to objects "chocolate", "rabbit" and "puppy"; assessors therefore tend to label sentences with TE-Pairs (she, chocolate), (she, rabbit), and (she, puppy) as positive emotions. However, S4 templates of the MAHs show that females are usually bitten and attacked by those objects. S1 and S2 templates represent that females do not often perform "love", "purchase" and other "pleasant" actions on those objects. Therefore, these TE-Pairs tend to match with "negative" RE-Pairs and result in wrong predictions.

\subsection{Context-sensitive problems vs. Emotion sensing}

Based on the aforementioned observations, incorrectly predicted sentences are resulted from "polysemy entities" and "semantic problems". Both situations can be concluded as "context-sensitive problems". For example, in many retrieved web pages, extracted sentences contain the polysemy entities "jaguar" and "giant" also frequently contain nearby sentences describing about "animals" and "adventures", respectively. As for the semantic problem, web page sentences mention "chocolate" are frequently relevant to party or politic events, such as "people are attacked by chocolates" in parties or politic activities. Many web pages also mention that puppies (or rabbit) 
probably attack (or bite) people during the playing activities. Therefore, the system collected these kinds of sentences for TE-Pairs (she, chocolate), (she, rabbit), and (she, puppy) and mined MAHs that are dominated by $\mathrm{S} 4$ templates. Consequently, these TE-Pairs are matched with RE-Pairs like (she, tiger) or (she, snake) and are predicted as "negative" emotions.

According to these observations, we conclude that emotions of sentences are context-sensitive. However, the context-sensitive problem is not considered in the tested sentences since each sentence is individually applied without nearby sentences. Generally speaking, emotion sensing applications should be domain-dependent so that the domain background knowledge facilitates the prediction of emotion. For example, applying the system to chatbot (chat robot) applications will obtain better performance of emotion sensing since previous typed sentences can be use to detect the background domain of the current chitchat, such as "animals", "adventures", or "parties". Therefore, domain-knowledge and the proposed emotion sensing system will be integrated to improve the system performance for various applications in the future.

\section{Conclusions and future works}

This paper proposes an event-level textual emotion detecting approach based on the common action distribution between event participants. Based on 11 RE-Pairs associated with 31 verb groups, sentences (parsed by the SRL tool) are collected from web search engines and manually labeled with emotions, Positive, Negative or Neutral. The web-based text mining approach is applied to collect and analyze large amount of event sentences as training and testing data. No need of any large-scale lexical sources or knowledgebase, the proposed system works well on predicting the emotion of a sentence, even the sentence contains entities or verbs that are absent in the knowledgebase or database. Experiment results indicate that the proposed system achieves a relative high precision about $85 \%$ in par with past studies. Also, the system is adaptive to the dynamic Web environment that novel name entities are frequently created in web pages.

As we concluded that the emotion sensing problem is context-sensitive, the future work is toward to applying the system to domain-dependent applications like chatbot. Based on the current result, improving the system for sensing more complicated sentences or emotions is the future research issue. In [Soong et al. 2001], machine learning techniques are applied to handle complicated sentences or paragraphs where many events are often intermingled together. For example, the sentence "I saw a snake chasing a frog" is simple but contains more than one event. Based on the 
emotion theory [Ortony et al. 1988], the emotion of a sentence is influenced by all participants contained in the sentence. As for sensing more complicated emotions, to enhance the usability of the system, the proposed method should be able to precisely sensing more emotional categories, such as big-six emotions [Ekman 1993], including Happy, Sadness, Fear, Anger, Disgust and Surprise. Those emotions are widely used in human-computer interactive applications.

\section{References}

[Bartneck 2002] C. Bartneck, "Integrating the OCC Model of Emotions in Embodied Characters," in Proceedings of the Workshop on Virtual Conversational Characters: Applications, Methods, and Research Challenges, 2002.

[Bates 1994] J. Bates, "The role of emotion in believable agents," Communications of the ACM, vol. 37 , no. 7, pp. 122-125, 1994.

[Carreras and Marquez 2005] X. Carreras and L. Marquez, "Introduction to the CoNLL-2005 Shared Task: Semantic Role Labeling," in Proceedings of Conference on Computational Natural Language Learning, 2005.

[Dyer 1987] M. Dyer, "Emotions and Their Computations: Three Computer Models," Cognition and Emotion, vol. 1, no. 3, pp. 323-347, 1987.

[Dang and Palmer 2005] H. Dang and M. Palmer, "The role of semantic roles in disambiguating verb senses," in Proceedings of Association for Computational Linguistics-05, 2005.

[Elliott 1992] C. Elliott, "The Affective Reasoner: A Process Model of Emotions in a Multi-agent System," Ph.D. dissertation, Northwestern University, The Institute for the Learning Sciences, Technical Report No. 32, 1992.

[Etzioni et al. 2005] O. Etzioni, M. Cafarella, and D. Downey, A. Popescu, T. Shaked, S. Soderland, D. S. Weld, and A. Yates, "Unsupervised named-entity extraction from the Web: an experimental study,” Artificial Intelligence, vol. 165, pp. 91-134, 2005.

[Ekman 1993] P. Ekman, "Facial Expression of Emotion,” American Psychologist, vol.48, pp. 384-392, 1993.

[Fellbaum 1998] C. Fellbaum, WordNet: A Lexical Database. The MIT Press, 1998.

[Girju et al. 2006] R. Girju, A. Badulescu, and D. Moldovan, "Automatic discovery of part-whole relations," Computational Linguistics, vol. 32, no. 1, pp. 83-135, 2006.

[Gildea and Jurafsky 2002] D. Gildea and D. Jurafsky, "Automatic Labeling of Semantic Roles," Computational Linguistics, vol.28, no. 3, pp. 245-288, 2002.

[Hearst 1992] M. Hearst, "Automatic Acquisition of Hyponyms from Large Text Corpora," in Proceedings of COLING, 1992.

[Koomen et al. 2005] P. Koomen, V. Punyakanok, D. Roth, and W. Yih, "Generalized Inference with Multiple Semantic Role Labeling System," in Proceedings of Conference on Computational Natural Language Learning, 2005.

[Kullback and Leibler 1951] S. Kullback and R.A. Leibler, "On information and sufficiency,” Annals of Mathematical Statistics, vol. 22, pp. 79-86, 1951. 
[Liu et al. 2003] H. Liu, H. Lieberman, and T. Selker, "A Model of Textual Affect Sensing using Real-World Knowledge," in Proceedings of the 2003 International Conference on Intelligent User Interfaces, 2003, pp. 125-132.

[Ortony et al. 1988] A. Ortony, G. Clore, and A. Collins, The cognitive structure of emotions. New York: Cambridge University Press, 1988.

[Picard 1997] R.W. Picard, Affective Computing, The MIT Press, Mass., 1997.

[Pantel and Pennacchiotti 2006] P. Pantel and M. Pennacchiotti, "Espresso: Leveraging generic patterns for automatically harvesting semantic relations," in Proceedings of the $2 I^{s t}$ Int. Conference on Computational Linguistics and the $44^{\text {th }}$ annual meeting of the Association for Computational Linguistics, 2006, pp. 113-120.

[Punyakanok et al. 2005] V. Punyakanok, D. Roth, and W. Yih, "The Necessity of Syntactic Parsing for Semantic Role Labeling," in Proceedings of the International Joint Conference on Artificial Intelligence, 2005, pp. 1117-1123.

[Pradhan et al. 2003] S. Pradhan, K. Hacioqlu, W. Ward, J.H. Martin, and D. Jurafsky, "Semantic role parsing: adding semantic structure to unstructure text," in IEEE International Conference on Data Mining, 2003, pp. 629-632.

[Pradhan et al. 2004] S. Pradhan, W. Hacioglu, J. Martin, and D. Jurafsky, "Shallow Semantic Parsing using Support Vector Machines," in Proceedings of the Human Language Technology Conference/North American chapter of the Association for Computational Linguistics annual meeting, 2004.

[Shaikh et al. 2007] Mostafa Shaikh, Helmut Prendinger, and Mitsuru Ishizuka, "SenseNet: A Linguistic Tool to Visualize Numerical-Valence based Sentiment of Textual Data," (poster paper) Proc 5th Int'l Conf on Natural Language Processing (ICON-07), Hyderabad, India, Jan. 2007, pp $147-152$.

[Soong et al. 2001] W.M. Soong, H.T. Ng, and D.C.Y. Lim, "A Machine Learning Approach to Coreference Resolution of Noun Phrases," Computational Linguistics, vol. 27, no. 4, pp. 521-544, 2001.

[Wu et al. 2006] C. Wu, Z. Chuang, and Y. Lin, "Emotion recognition from text using semantic labels and separable mixture models," ACM Transactions on Asian Language Information Processing, vol. 5, no. 2, pp. 165-183, 2006. 
Appendix 1: Synonym groups for the verbs

Group

\begin{tabular}{l}
\hline 1 \\
\hline 2 \\
\hline 3 \\
\hline 4 \\
\hline 5 \\
\hline 7 \\
\hline 8 \\
\hline 9 \\
\hline 10 \\
\hline 11 \\
\hline 12 \\
\hline 13 \\
\hline 14 \\
\hline 16 \\
\hline 17 \\
\hline 18 \\
\hline 20 \\
\hline 21
\end{tabular}

22

23

24

25

26

27

28

29

30

31

32

33

34

35 36

37

38

39

40

41

42

43

44

45

46

47

\begin{tabular}{l}
\hline 48 \\
\hline 49 \\
\hline 50 \\
\hline 51
\end{tabular}

\begin{tabular}{l}
\hline 52 \\
\hline 53 \\
\hline 54 \\
\hline 55 \\
\hline 56 \\
\hline 57
\end{tabular}

\section{Verbs}

Abandon (cease, discard, terminate, discontinue, stop, halt, escape, restrain, quit, forsake, leave)

Abduct (kidnap, hijack)

Abound (teem, swarm, flourish, overflow)

Accept (allow, permit, admit, recognize, adopt, agree, approve, accede, obey)

Access (entry, approach)

Addict (affect)

Aim (try, intend, attempt, attend, direct, point)

Ask (question, inquire, query, interrogate, request, beg, solicit, demand, plead, consult)

Astonish (surprise, amaze, astound, shock)

Attach (affix, tack, append, adhere)

Attack (hurt, beset, hit, cuff, assail, bombard, kick, strike, swat, punch, assault, maul)

Avenge (revenge, retaliate, requite, punish, repay, retort)

Award (reward, prize, medal, trophy, grant, gift, bestow)

Banish (exile, deport, outlaw)

Bark (yelp, yip, yap, bay, woof, ululate, bowwow, snap, hoot)

Bear (tolerate, yield, endure)

Begin (start ,commence)

Bite (pierce, nip)

Blame (berate, accuse, impeach, reprehend, decry, condemn, denounce, charge, indict)

Bother (disturb, annoy, irritate)

Challenge (confront, question, dispute, counteract, defy, doubt, dare, suspect, protest, gage, controvert, mistrust, withdraw, struggle)

Change (alter, vary, deviate, substitute, replace, modify, convert, shift, switch, transfer, turn, exchange, transform)

Clarify (explain, refine, simplify, illuminate, illustrate, expound, specify, purify, define, interpret, unfold)

Classify (organize, categorize, sort, catalog, assort, grade, file, rank, collocate)

Collect (gather, assemble, accumulate)

Compete (contest, rival, contend, fight, strive)

Conduct (manage, direct, guide, control, govern, captain, command, instruct, steer)

Congratulate (bless, compliment, flatter, commend, praise)

Conquer (prevail, overtake, vanquish, defeat, overcome, rout, overpower)

Contain (include, hold, comprise, involve, enclose)

Cooperate (coact, coordinate, conspire)

Compare (correlate, parallel)

Create (build, make, manufacture, fabricate, develop, form, compose, shape, devise, invent, originate)

Dance (dance, rock, reel, twirl, caper, step, swing, prance, disco, hop, gambol, romp, leap, wiggle, wriggle)

Decrease (decline, decelerate, detract, devalue, dilute, weaken, thin, diminish, deduct, deteriorate, demote, degrade, reduce, lower, debase, abase, cheapen, devaluate)

Deliver (consign, convey, transport, transmit, send, dispatch, forward, pass)

Determine (decide, judge)

Devote(devote, consecrate, hallow)

Die (decease, perish, expire)

Disguise (conceal, hide, camouflage, misrepresent)

Ease (facilitate, relieve, help, comfort, relax, loosen, aid, lighten, assuage, allay, release)

Eat (dine, chew, swallow, feed, graze, devour, nibble, gobble, gulp)

Emphasize (stress, punctuate, accent, mark, intensify, highlight,

underscore, accentuate,

Encourage (support, urge, sponsor, promote, cheer, inspire, boost, excite, arouse, awaken, wake, kindle, pique, provoke, actuate, rouse, arise, aspire)

Entertain (play, amuse, delight, titillate, tickle, please, show)

Espouse (espouse, betroth)

Evaluate (valuate, assess, compute, count, account, calculate, gauge, rate, measure, criticize, weigh, reckon, appraise, approximate)

Evolve (breed, develop, diffuse, grow, )

Examine (inspect, observe, study, review, investigate, scrutinize, diagnose)

Expect (anticipate, await, hope)

Express (tell, present, describe, allege , announce, declare, acquaint, articulate, aver, inform, mean, signify, connote, perform, talk, assert, pronounce, confide, denote, detail, elaborate, display, show, speak, appear, say, show)

Fall (immerse, drop, plunge, swoop, sink)

Find (discover, detect, excavate, dredge, dig, unearth, dissect, learn, ascertain, locate)

Finish (accomplish, fulfill, complete, consummate, end, terminate, conclude, cease, stop)

Force (compel, pressure, compress, confine, bend, constrict, tighten, lessen, press, push, brake, quell, choke, coerce, oblige, motivate, propel)

Forget (disregard, overlook, ignore, pretermit, neglect)

Frustrate (dampen, dishearten, depress, despair, discourage, dissuade, dismay, thwart) 
71

72

73

74

75

76

77

78

\begin{tabular}{l}
\hline 79 \\
\hline 80 \\
\hline 81 \\
\hline 82
\end{tabular}

\begin{tabular}{l}
\hline 83 \\
\hline 84 \\
\hline 85 \\
\hline 86
\end{tabular}

87

Gain (earn, secure, obtain, acquire, receive, get)

Go (run, walk)

Greet (welcome, invite)

Group (flock, sort, classify, organize, arrange, assemble, cluster, gather)

Grow (breed, develop, diffuse, disseminate, grow, evolve, mature, raise)

Guarantee (affirm, guarantee, assure, convince, pledge, insure, satisfy, vow, vouch, ensure, promise, attest, certify, depose, testify, confirm, verify, validate, authenticate, corroborate, substantiate, warrant, evidence, endorse, prove, cite, commit)

Hate (dislike, despise, abominate, aggravate, infuriate, anger, grumble, complain, argue, quarrel, detest, disapprove, disfavor, oppose, object, disparage, belittle, underestimate, dispute, dissatisfy, displease, exacerbate, envenom, embitter)

Hide (hide, cover, conceal, blanket, bury)

Increase (enlarge, extend, expand, augment, multiply, advance, raise, add, annex, amplify, greaten, widen, broaden, lengthen, elongate, prolong, prolongate, protract, dilate, ascend, deepen, strengthen, reinforce, redouble, heighten, rise, mount, rocket)

Kill (destroy, kill, slay, slaughter, murder)

Lean (bend, lean, slope, slant, incline, rest)

Lie (beguile, lie, betray, deceive, misdirect, misinform, fool, delude, hoax, dupe, chisel, defraud, disbelieve, discredit, distrust, fib, falsify)

Live (exist, dwell, inhabit)

Love (like, enjoy, cherish, treasure, adore, admire, appreciate, relish, fancy, idolize)

Marry (marry, wed)

Meet (encounter, join, see, incorporate, unite, connect, converge, confront)

Move(lift, carry, send, throw, push, pull)

Name( label, title, term, tag, identify)

Open (launch, expose, uncover, disclose, disclose)

Plan ( propose, design, plot, arrange, program, patch, project, scheme)

Pollute (defile, contaminate, infect, taint, tarnish, foul, poison, alloy, smoke, deteriorate, mess, demoralize, spoil)

Prepare (equip, concoct, provide, ready)

Purchase (buy, shop, purchase, order, market)

Put (place, fix, lay, set, deposit, arrange, spread)

Remove (avert, delete, erase, cancel, obliterate, denude, disappear, disband, remove, withdraw, extract, eject, expel, oust, evacuate, eradicate, dethrone, unseat, scatter, dislodge, dismantle, dispel, replace, displace)

Ride (cruise, drive, journey)

Save (preserve, keep, guard, maintain, store, reserve, scrimp, conserve)

Want (wish, crave, need, require, covet, desire, fancy, demand, ambition)

Warn (threaten, intimidate, bulldoze, alert, admonish, menace, browbeat, terrorize, thunder, horrify, scare,

frighten, appall, deter, bully, fear, startle, terrify)

Worry (concern, care) 
Appendix 2: Emotion rules for the reference entity pair with "she" as the subject and "diamond ring" as the object.

\begin{tabular}{|c|c|c|}
\hline 1 & Abandon & Unhappy \\
\hline 2 & Abduct & N/A \\
\hline 3 & Abound & N/A \\
\hline 4 & Accept & Happy \\
\hline 5 & Access & Happy \\
\hline 6 & Addict & Happy \\
\hline 7 & Aim & N/A \\
\hline 8 & Ask & Neutral \\
\hline 9 & Astonish & Happy \\
\hline 10 & Attach & Happy \\
\hline 11 & Attack & Unhappy \\
\hline 12 & Avenge & N/A \\
\hline 13 & Award & Happy \\
\hline 14 & Banish & N/A \\
\hline 15 & Bark & Unhappy \\
\hline 16 & Bear & N/A \\
\hline 17 & Begin & N/A \\
\hline 18 & Bite & Unhappy \\
\hline 19 & Blame & Unhappy \\
\hline 20 & Bother & N/A \\
\hline 21 & Challenge & N/A \\
\hline 22 & Change & Happy \\
\hline 23 & Clarify & Happy \\
\hline 24 & Classify & Happy \\
\hline 25 & Collect & Happy \\
\hline 26 & Conduct & N/A \\
\hline 27 & Compete & Unhappy \\
\hline 28 & Congratulate & N/A \\
\hline 29 & Conquer & N/A \\
\hline 30 & Contain & Happy \\
\hline 31 & Cooperate & N/A \\
\hline 32 & Compare & Neutral \\
\hline 33 & Create & Happy \\
\hline 34 & Dance & N/A \\
\hline 35 & Decrease & Unhappy \\
\hline 36 & Deliver & Neutral \\
\hline 37 & Determine & Neutral \\
\hline 38 & Devote & N/A \\
\hline 39 & Die & Unhappy \\
\hline 40 & Disguise & N/A \\
\hline 41 & Ease & N/A \\
\hline 42 & Eat & Unhappy \\
\hline 43 & Emphasize & Happy \\
\hline 44 & Encourage & N/A \\
\hline
\end{tabular}

\begin{tabular}{|c|c|c|}
\hline 45 & Entertain & Happy \\
\hline 46 & Espouse & Happy \\
\hline 47 & Evaluate & Neutral \\
\hline 48 & Evolve & Happy \\
\hline 49 & Examine & Neutral \\
\hline 50 & Expect & Happy \\
\hline 51 & Express & Happy \\
\hline 52 & Fall & Unhappy \\
\hline 53 & Find & Happy \\
\hline 54 & Finish & Unhappy \\
\hline 55 & Force & Unhappy \\
\hline 56 & Forget & Unhappy \\
\hline 57 & Frustrate & N/A \\
\hline 58 & Gain & Happy \\
\hline 59 & Go & N/A \\
\hline 60 & Greet & Happy \\
\hline 61 & Group & Happy \\
\hline 62 & Grow & N/A \\
\hline 63 & Shout & Unhappy \\
\hline 64 & Guarantee & N/A \\
\hline 65 & Hate & Unhappy \\
\hline 66 & Hide & Unhappy \\
\hline 67 & Kill & Unhappy \\
\hline 68 & Lean & Happy \\
\hline 69 & Lie & N/A \\
\hline 70 & Live & N/A \\
\hline 71 & Love & Happy \\
\hline 72 & Marry & N/A \\
\hline 73 & Meet & Happy \\
\hline 74 & Move & Neutral \\
\hline 75 & Name & Neutral \\
\hline 76 & Open & Happy \\
\hline 77 & Plan & N/A \\
\hline 78 & Pollute & Unhappy \\
\hline 79 & Prepare & Happy \\
\hline 80 & Purchase & Happy \\
\hline 81 & Put & Happy \\
\hline 82 & Remove & Unhappy \\
\hline 83 & Ride & Neutral \\
\hline 84 & Save & Happy \\
\hline 85 & Want & Happy \\
\hline 86 & Warn & N/A \\
\hline 87 & Worry & Unhappy \\
\hline
\end{tabular}




\begin{tabular}{|c|c|c|c|c|c|c|c|}
\hline Verb & Object & Subjective & System & Verb & Object & Subjective & System \\
\hline get & chocolate & Happy & Unhappy & lose & scholarship & Unhappy & Unhappy \\
\hline award & chocolate & Happy & Unhappy & reduce & scholarship & Unhappy & Unhappy \\
\hline order & puppy & Happy & Unhappy & discard & skirt & Unhappy & Unhappy \\
\hline see & puppy & Happy & Unhappy & discard & vaio & Unhappy & Unhappy \\
\hline buy & rabbit & Happy & Unhappy & misplace & vaio & Unhappy & Unhappy \\
\hline receive & dessert & Happy & Happy & lean & cobra & Unhappy & Unhappy \\
\hline receive & diamond & Happy & Happy & hate & crocodile & Unhappy & Unhappy \\
\hline see & diamond & Happy & Happy & see & ghost & Unhappy & Happy \\
\hline get & wii & Happy & Happy & meet & ghost & Unhappy & Happy \\
\hline receive & golden ring & Happy & Happy & meet & snake & Unhappy & Unhappy \\
\hline receive & iphone & Happy & Happy & find & snake & Unhappy & Unhappy \\
\hline get & jewel & Happy & Happy & detect & vampire & Unhappy & Unhappy \\
\hline receive & jewel & Happy & Happy & see & vampire & Unhappy & Unhappy \\
\hline award & luxury yacht & Happy & Happy & meet & vampire & Unhappy & Unhappy \\
\hline buy & luxury yacht & Happy & Happy & look & vicious & Unhappy & Unhappy \\
\hline purchase & miniskirt & Happy & Happy & see & vicious & Unhappy & Unhappy \\
\hline wish & ipod nano & Happy & Happy & meet & vicious & Unhappy & Unhappy \\
\hline see & new camera & Happy & Happy & hate & centipede & Unhappy & Unhappy \\
\hline reward & new house & Happy & Happy & detect & centipede & Unhappy & Unhappy \\
\hline desire & new scooter & Happy & Happy & look & rattlesnake & Unhappy & Unhappy \\
\hline accept & perfume & Happy & Happy & see & rattlesnake & Unhappy & Unhappy \\
\hline wear & perfume & Happy & Happy & see & zombie & Unhappy & Unhappy \\
\hline buy & perfume & Happy & Happy & look & zombie & Unhappy & Unhappy \\
\hline receive & prada & Happy & Happy & find & gang & Unhappy & Happy \\
\hline desire & prada & Happy & Happy & see & demon & Unhappy & Unhappy \\
\hline award & prize & Happy & Unhappy & see & rat & Unhappy & Unhappy \\
\hline accept & prize & Happy & Unhappy & find & rat & Unhappy & Unhappy \\
\hline accept & ps2 & Happy & Happy & lean & rat & Unhappy & Unhappy \\
\hline get & ps2 & Happy & Happy & detect & bobcat & Unhappy & Happy \\
\hline receive & rolex & Happy & Happy & meet & villain & Unhappy & Unhappy \\
\hline purchase & rolex & Happy & Happy & see & villain & Unhappy & Unhappy \\
\hline get & scholarship & Happy & Happy & eat & apple & Neutral & Neutral \\
\hline receive & scholarship & Happy & Happy & change & hair & Neutral & Neutral \\
\hline award & tivo & Happy & Happy & express & hair & Neutral & Neutral \\
\hline buy & tivo & Happy & Happy & show & hair & Neutral & Neutral \\
\hline join & tour & Happy & Unhappy & find & desk & Neutral & Neutral \\
\hline wish & tour & Happy & Unhappy & receive & desk & Neutral & Neutral \\
\hline see & vaio & Happy & Happy & purchase & door & Neutral & Neutral \\
\hline desire & watch & Happy & Happy & See & Wine & Neutral & Neutral \\
\hline buy & watch & Happy & Happy & award & window & Neutral & Neutral \\
\hline punch & robber & Happy & Happy & receive & window & Neutral & Neutral \\
\hline kill & malady & Happy & Unhappy & receive & coin & Neutral & Neutral \\
\hline shoot & malady & Happy & Unhappy & Get & coin & Neutral & Neutral \\
\hline kill & rat & Happy & Happy & receive & spoon & Neutral & Neutral \\
\hline kick & rat & Happy & Happy & award & spoon & Neutral & Neutral \\
\hline attack & rat & Happy & Happy & award & toothbrush & Neutral & Neutral \\
\hline lose & benz & Unhappy & Unhappy & accept & toothbrush & Neutral & Neutral \\
\hline destroy & benz & Unhappy & Unhappy & get & soap & Neutral & Neutral \\
\hline break & bikini & Unhappy & Unhappy & award & soap & Neutral & Neutral \\
\hline shatter & bikini & Unhappy & Unhappy & receive & soap & Neutral & Neutral \\
\hline lose & bonus & Unhappy & Unhappy & see & fork & Neutral & Neutral \\
\hline abandon & bonus & Unhappy & Unhappy & get & fork & Neutral & Neutral \\
\hline destroy & iphone & Unhappy & Unhappy & receive & fork & Neutral & Neutral \\
\hline break & iphone & Unhappy & Unhappy & receive & curtain & Neutral & Neutral \\
\hline abandon & jewel & Unhappy & Unhappy & receive & wheat & Neutral & Neutral \\
\hline lose & jewel & Unhappy & Unhappy & purchase & wheat & Neutral & Neutral \\
\hline lose & lv & Unhappy & Unhappy & open & document & Neutral & Neutral \\
\hline shatter & lv & Unhappy & Unhappy & touch & hair & Neutral & Neutral \\
\hline dislike & miniskirt & Unhappy & Unhappy & close & envelope & Neutral & Neutral \\
\hline lose & miniskirt & Unhappy & Unhappy & accept & envelope & Neutral & Neutral \\
\hline destroy & ipod nano & Unhappy & Unhappy & cut & hair & Neutral & Neutral \\
\hline break & new scooter & Unhappy & Unhappy & lose & cotton tag & Neutral & Unhappy \\
\hline shatter & new scooter & Unhappy & Unhappy & open & envelope & Neutral & Neutral \\
\hline lose & newborn baby & Unhappy & Unhappy & receive & envelope & Neutral & Neutral \\
\hline abandon & newborn baby & Unhappy & Unhappy & buy & envelope & Neutral & Neutral \\
\hline break & prada & Unhappy & Unhappy & get & envelope & Neutral & Neutral \\
\hline
\end{tabular}




\begin{tabular}{|c|c|c|c|c|c|c|c|}
\hline abandon & prada & Unhappy & Unhappy & see & desk & Neutral & Neutral \\
\hline lose & prize & Unhappy & Unhappy & award & desk & Neutral & Neutral \\
\hline kill & prize & Unhappy & Unhappy & take & toothbrush & Neutral & Neutral \\
\hline reduce & prize & Unhappy & Unhappy & eat & wheat & Neutral & Neutral \\
\hline shatter & rolex & Unhappy & Unhappy & buy & wheat & Neutral & Neutral \\
\hline dislike & rolex & Unhappy & Unhappy & order & wheat & Neutral & Neutral \\
\hline open & door & Neutral & Neutral & touch & window & Neutral & Neutral \\
\hline receive & door & Neutral & Neutral & get & window & Neutral & Neutral \\
\hline see & wheat & Neutral & Neutral & find & window & Neutral & Neutral \\
\hline get & chair & Neutral & Neutral & get & desk & Neutral & Neutral \\
\hline receive & chair & Neutral & Neutral & find & toothbrush & Neutral & Neutral \\
\hline receive & shop & Neutral & Neutral & touch & desk & Neutral & Neutral \\
\hline arrive & train station & Neutral & Neutral & buy & toothbrush & Neutral & Neutral \\
\hline look & train station & Neutral & Neutral & purchase & toothbrush & Neutral & Neutral \\
\hline meet & train station & Neutral & Neutral & & & & \\
\hline
\end{tabular}

